\title{
Identification of Optical Transitions from CdTe and CdMnTe Quantum Dots Embedded in ZnTe Nanowires
}

\author{
M. Szymura, Ł. KŁopotowski, P. Wojnar, G. Karczewski, T. Wojtowicz \\ AND J. KossuT
}

Institute of Physics, Polish Academy of Sciences, al. Lotników 32/46, 02-668 Warsaw, Poland

\begin{abstract}
We study photoluminescence properties of CdTe and CdMnTe quantum dots embedded in ZnTe nanowires. The nanowires were grown by molecular beam epitaxy, applying the vapor-liquid-solid growth mechanism. Linear polarization anisotropy measurements allow us to assess that the excitonic transitions originate from a single nanowire. We identify the optical transitions by comparing observed spectroscopic shifts with the universal emission pattern from the epitaxial CdTe dots. We support this identification by analyzing the photoluminescence intensity dependence on excitation power.
\end{abstract}

DOI: 10.12693/APhysPolA.124.824

PACS: 78.55.Et, 78.67.Uh, 78.67.Hc

\section{Introduction}

The recent interest in semiconductor nanowires (NWs) is largely fueled by their optical properties. Single NWs exhibit a large polarization anisotropy of both light excitation and emission [1] — useful in developing polarization sensitive optical elements. Moreover, NWs offer a significantly increased collection of light from an embedded light source such as a quantum dot (QD) [2]. Therefore, NWs containing QDs are promising building blocks of future nanophotonic devices. Indeed, single photon emission from such nanostructures was reported $[3,4]$. Moreover, controllable charging of a QD embedded in a NW was demonstrated, paving the way towards their applications in quantum information processing [5].

In this report, we investigated photoluminescence (PL) properties of CdTe and CdMnTe dots grown as insertions in ZnTe NWs. In such dots grown epitaxially, recombinations of various excitonic complexes are known to exhibit a universal sequence in energy [6, 7]. We compared the PL spectra of the NW dots to their epitaxial counterparts and perform a preliminary identification of the transitions investigating the intensity dependence on excitation power.

\section{Samples and experiment}

Two samples are investigated: $\mathrm{ZnTe}$ nanowires with a single CdTe quantum dot, grown on GaAs substrate and ZnTe nanowires with two dots: made of $\mathrm{CdTe}$ and CdMnTe, grown on $\mathrm{Si}$ substrate. The nanowires were grown by the gold nanocatalysts assisted molecular beam epitaxy by applying the vapor-liquid-solid growth mechanism [8]. Nanometer sized droplets of gold eutectic were formed from a $1 \mathrm{~nm}$ thick gold layer deposited on (111) oriented GaAs or Si substrate. The details of the growth procedure can be found in Ref. [9]. QD size was controlled by the growth time and temperature. For NWs with a single QD, $\mathrm{Cd}$ and Te effusion cells were opened for $60 \mathrm{~s}$. In the two-dot sample, the CdTe and CdMnTe QDs and the ZnTe spacer layer were all grown for $120 \mathrm{~s}$. Mn concentration in CdMnTe QDs was about $1 \%$. The morphology of the NWs was examined by scanning electron microscopy (SEM). Figure 1a shows a typical SEM image of an as-grown sample containing $\mathrm{ZnTe}$ nanowires with CdTe and CdMnTe dots. The NW diameter ranged from $30 \mathrm{~nm}$ to $150 \mathrm{~nm}$ and the length was up to $1.8 \mu \mathrm{m}$. NW density was about 125 nanowires $/ \mu \mathrm{m}^{2}$. The NWs on GaAs grew along the $\langle 111\rangle$ axis. On the other hand, the growth on Si occurred on an amorphous oxidized silicon layer which resulted in NW growth in arbitrary directions.

In order to investigate the PL from single NWs, we ultrasonically removed the as-grown wires from the substrate and dispersed them onto a $\mathrm{Si}$ wafer. Figure $1 \mathrm{~b}$ shows a SEM image of a typical single NW with the gold droplet on top.
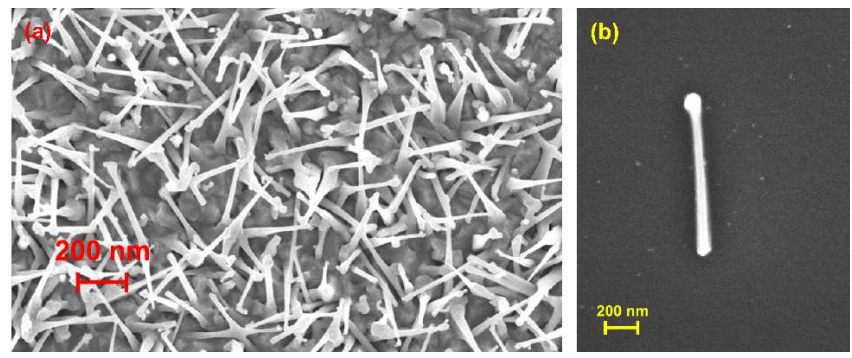

Fig. 1. (a) SEM image of an as-grown sample containing ZnTe NWs with CdTe and CdMnTe QDs. (b) SEM image of a single nanowire removed from the as-grown sample.

We measured PL excited with $473 \mathrm{~nm}$ laser. In order to collect light from a single nanostructure, the laser beam was focused onto $a \approx 2 \mu \mathrm{m}$ spot with a microscope objective. The signal passed through a rotatable half-wave plate and a fixed linear polarizer allowing the stud- 
ies of polarization anisotropy. All measurements were performed at $10 \mathrm{~K}$.

\section{Results and discussion}

Initial PL measurements were performed for a relatively low excitation power of $100 \mu \mathrm{W}$ in order to avoid the appearance of multi-excitonic complexes. For the sample with one CdTe QD in a NW, at low excitation power we observe a single transition, with an average linewidth of about $2 \mathrm{meV}$ (see Fig. 2a). This value is about 10 times larger than for CdTe QDs grown epitaxially $[6,7]$. With increasing excitation power two additional lines appear, redshifted by roughly $5 \mathrm{meV}$ and $8 \mathrm{meV}$ with respect to the formerly observed transition (see Fig. 2a). In order to prove that the observed transitions originate from the same NW, we performed measurements of the linear polarization anisotropy. We observe periodic dependence of PL intensity on the analyzer angle (see Fig. 2b). Polarization anisotropy is caused by the dielectric mismatch between the NW and the surrounding vacuum. The mismatch causes strong suppression of the component of the electric field inside and perpendicular to the NW [1]. Therefore, the maximum PL intensity, $I_{\max }$ (minimum, $I_{\min }$ ) corresponds to the linear polarization oriented parallel (perpendicular) to the nanowire axis. Importantly, the maximum and minimum PL intensities are observed for the same analyzer angles for all transitions (see Fig. 2b). The polarization degree, defined as $P=\left(I_{\max }-I_{\min }\right) /\left(I_{\max }+I_{\min }\right)$, is evaluated by fitting to the intensity dependence on the analyzer angle a square of sine function (see Fig. 2b) plus a temporal intensity drift related to the mechanical instability of the setup. The values obtained for the three observed transitions are $77 \%, 81 \%$, and $90 \%$ (see Fig. 2b). Since for the observed transitions the anisotropy angles and the polarization degrees are the same within the accuracy of the measurement, we conclude that they originate from the same nanowire.

We tentatively identify the transitions by comparing the spectroscopic shifts with those observed for epitaxial CdTe dots [6, 7]. Thus, we ascribe the transition redshifted by $5 \mathrm{meV}$ to recombination of a charged exciton (CX), and the transition redshifted by $8 \mathrm{meV}$ to the biexciton $(2 \mathrm{X})$. We also remark that an analogous transition sequence was observed in the initial studies of CdTe QDs embedded in ZnTe NWs [9]. In epitaxial CdTe dots, usually both $\mathrm{CX}$ transitions $\left(\mathrm{X}^{+}\right.$and $\mathrm{X}^{-}$) are observed. Due to the increased linewidth, we are unable to clearly resolve them in our case.

PL measurements as a function of the excitation power allow us to support the above transition identification. We fit power law functions $I \sim P^{\alpha}$ to the dependence of the PL intensities on excitation power $P$ (see Fig. $2 \mathrm{c}-\mathrm{e}$ ). The fitted exponents $\alpha$ are equal to $1.2 \pm 0.2$ for $\mathrm{X}$, $1.5 \pm 0.2$ for $\mathrm{CX}, 2.1 \pm 0.2$ for $2 \mathrm{X}$. These values, within the experimental error, reflect the linear and quadratic increase expected for the $\mathrm{X}$ and $2 \mathrm{X}$, respectively. For
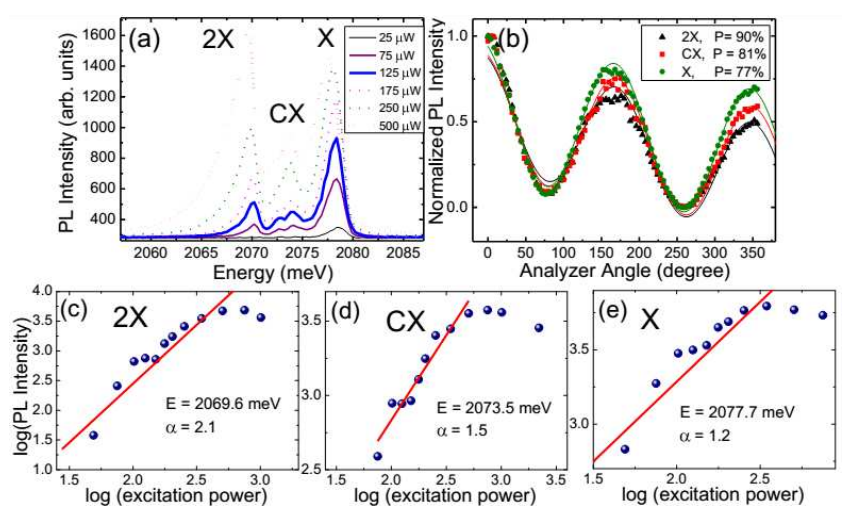

Fig. 2. (a) PL spectra measured for various excitation powers on a single NW containing a CdTe QD. (b) Normalized PL intensity of emission line as a function of analyzer angle for $2 \mathrm{X}, \mathrm{CX}, \mathrm{X}$, the lines represented fit square of the sine function. (c, d,e) Transition intensity as a function of excitation power for $2 \mathrm{X}, \mathrm{CX}$, and $\mathrm{X}$.

the $\mathrm{CX}$, we anticipate an intermediate value of $\alpha$, since excitation of a $\mathrm{CX}$ requires capturing three carriers.

The $\mathrm{CX}$ and $2 \mathrm{X}$ transitions appear on average 6 and $10 \mathrm{meV}$ below the $\mathrm{X}$ transition, respectively, while for epitaxial CdTe QDs, the average spectroscopic shifts for the $\mathrm{X}^{+}, \mathrm{X}^{-}$, and the $2 \mathrm{X}$ are equal to $7,10,13 \mathrm{meV}$, respectively. Explanation of the smaller spectroscopic shifts for QDs embedded in NWs lies beyond the scope of this paper and requires studies of the Coulomb interactions in these nanostructures.

For the NWs with two QDs, CdTe and CdMnTe, in the PL spectrum we observe two types of lines: (i) transitions with an average linewidth of about $2 \mathrm{meV}$, which we identify as emissions from CdTe QDs and (ii) much broader spectral structures with a width of about $7 \mathrm{meV}$. We identify these transitions as the emission from CdMnTe QDs. Indeed, presence of Mn ions causes a broadening of the transitions due to magnetization fluctuations. We do not observe transitions from both the CdTe and CdMnTe QDs from the same NW. This may be caused by the process of isolating the individual wires - during the sonication they can break between the two QDs.

Another explanation involves a tunnel coupling between the QDs. As a result of this coupling, the carriers are transferred to the lower energy dot and thus the emission is observed always from only one of them. The plausibility of the two explanations depends crucially on the width of the spacer layer between the dots. Unfortunately, it is difficult to determine this width solely from the growth parameters. Therefore, high resolution transmission electron microscopy studies are required to explain the lack of both emissions from a single NW.

Figure 3 shows PL spectra for a CdMnTe QD measured for various excitation powers. As stated above, at low powers a single feature is observed. With increasing excitation power, the intensity at the low energy side of this feature clearly grows quicker than the high energy 
side. This behavior is qualitatively similar to the one observed for the CdTe dot (see Fig. 2a), where the intensity of the redshifted transitions, $\mathrm{CX}$ and $2 \mathrm{X}$, increased faster than the intensity of the X. However, the fluctuation-induced broadening of the transitions, preclude precise identification of lines.

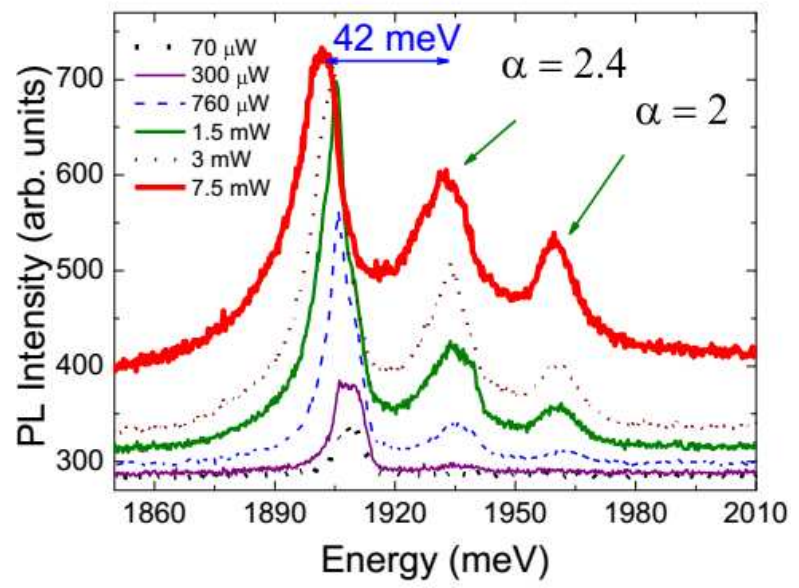

Fig. 3. PL intensity as a function of excitation power for NW with CdMnTe QD. $\alpha$ is an exponent obtained by fitting of a power law to the measured dependences.

As the excitation power is increased beyond $0.5 \mathrm{~mW}$, additional lines appear, which are blueshifted by about $42 \mathrm{meV}$ with respect to the main line. The intensities of these lines increase superlinearly with the excitation power. Therefore, we suggest that these lines originate from transitions from the $p$-shell.

\section{Conclusions}

ZnTe NWs with single CdTe QDs or with two QDs: $\mathrm{CdTe}$ and CdMnTe have been investigated. NWs were grown by molecular beam epitaxy using vapor-liquid-solid growth mechanism. We have proposed identification of optical transitions from QDs based on the spectral position in the PL spectrum. Moreover, we have supported our identification with the analysis of the PL intensity dependence on excitation power. We have identified the recombinations of the exciton, charged exciton and biexciton. For CdMnTe QDs we have observed features most probably related to recombinations from the $p$-shell.

\section{Acknowledgments}

This research was supported by Polish Ministry of Science and Higher Education NCN grant No. 2011/01/B/ ST3/02287.

\section{References}

[1] J. Wang, M.S. Gudiksen, X. Duan, Y. Cui, C.M. Lieber, Science 293, 1455 (2001).

[2] J. Claudon, J. Bleuse, N.S. Malik, M. Bazin, P. Jaffrennou, N. Gregersen, Ch. Sauvan, P. Lalanne, J.M. Gérard, Nature Photon. 4, 174 (2010).

[3] M.T. Borgstrom, V. Zwiller, E. Muller, A. Imamoglu, Nano Lett. 5, 1439 (2005).

[4] G. Sallen, A. Tribu, T. Aichele, R. André, L. Besombes, C. Bougerol, M. Richard, S. Tatarenko, K. Kheng, J.-Ph. Poizat, Phys. Rev. Lett. B 80, 085310 (2009).

[5] M. van Kouwen, M.E. Reimer, A.W. Hidma, M.H.M. van Weert, R.E. Algra, E.P.A.M. Bakkers, L.P. Kouwenhoven, V. Zwiller, Nano Lett. 10, 1817 (2010).

[6] Ł. Kłopotowski, V. Voliotis, A. Kudelski, A.I. Tartakovskii, P. Wojnar, K. Fronc, R. Grousson, O. Krebs, M.S. Skolnick, G. Karczewski, T. Wojtowicz, Phys. Rev. B 83, 155319 (2011).

[7] T. Kazimierczuk, T. Smolenski, M. Goryca, Ł. Kłopotowski, P. Wojnar, K. Fronc, A. Golnik, M. Nawrocki, J.A. Gaj, P. Kossacki, Phys. Rev. B 84, 165319 (2011).

[8] S. Wagner, W.C. Ellis, Appl. Phys. Lett. 4, 89 (1964).

[9] P. Wojnar, E. Janik, L.T. Baczewski, S. Kret, G. Karczewski, T. Wojtowicz, M. Goryca, T. Kazimierczuk, P. Kossacki, Appl. Phys. Lett. 99, 113109 (2011). 